Table S1. Oligonucleotide primers used in this study

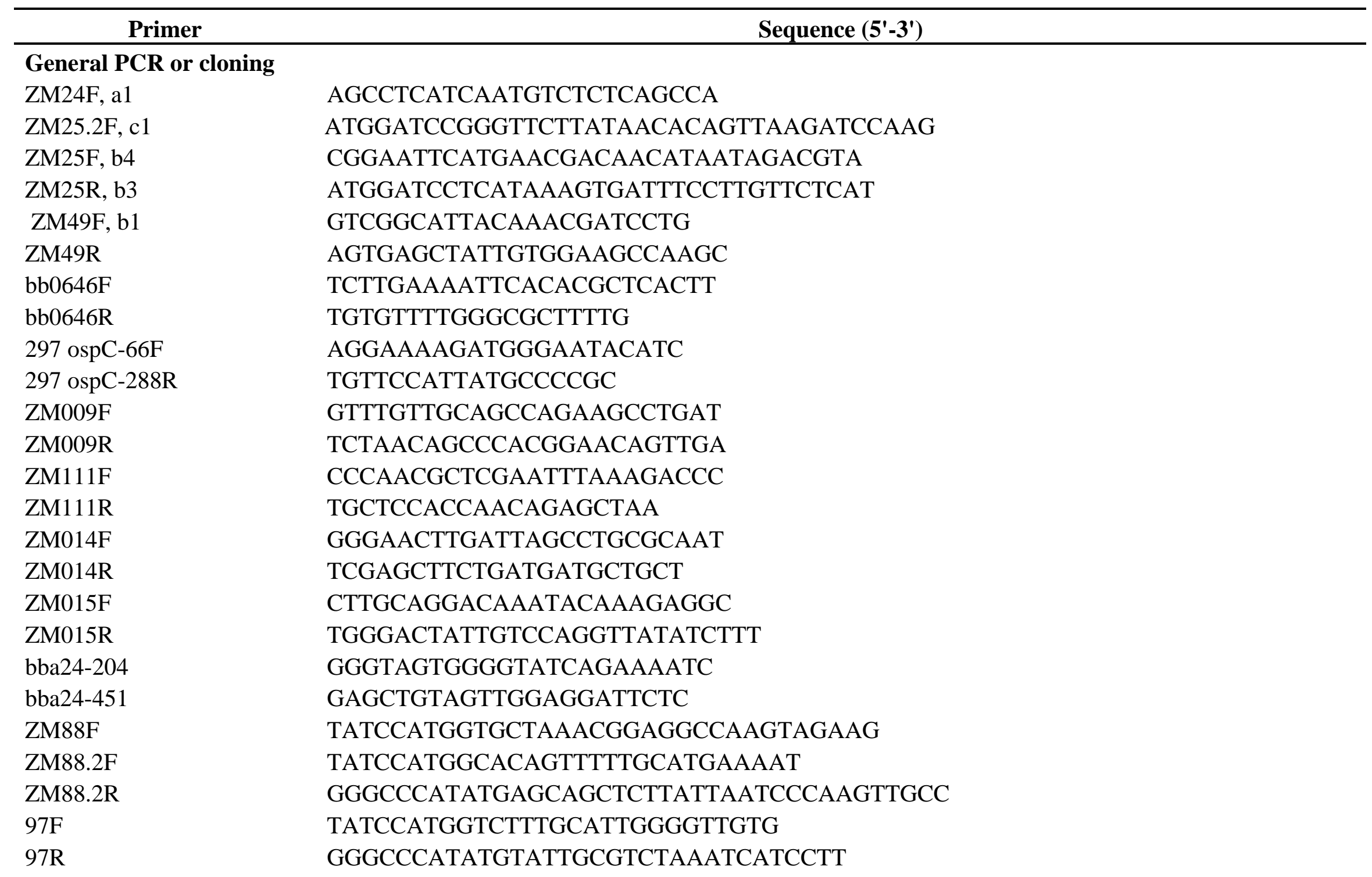


ZM107F

ZM108R

ZM57

ZM61

ZM69F

ZM69R

\section{For EMSA}

ZM126F

ZM126R

ZM127F

ZM127R

ZM132F

ZM132R

ZM147F

ZM147R

ZM149F

ZM149R

ZM157F

ZM157R

ZM160F

ZM160R

ZM161F

ZM161R

ZM166F

ZM166R

ZM155F

ZM155R

ZM156F
TATCCATGGCCTGAGTATTCATTATATAAGTC

GCGCATATGTAATTTGTGCCTCCTTTTTATTTATG

AGATCTATTTTATTTTATTTTTCATAAAGTGGGCTAAA

CATATGCTTTTCCCGTGGCTTCTTTT

ATGAACGACAACATAATAGACGTA

TCATAAAGTGATTTCCTTGTTCTCAT

TTGCATGGAAATTAAGTAGTAAAAACTTAATCACAATATTCAAGAAAGGGGAGAAAATAT ATATTTTCTCCCCTTTCTTGAATATTGTGATTAAGTTTTTACTACTTAATTTCCATGCAA TTTAGTTTAAAACCATTTTTAAATTAAATTGGCACAGTTTTTGCATGAAAATTAAGTAGT ACTACTTAATTTTCATGCAAAAACTGTGCCAATTTAATTTAAAAATGGTTTTAAACTAAA GGCCTTGCCGATTTAATTTACAATCAATTACAAAAAAGTAAATAATTCAAAAAATACTCC GGAGTATTTTTTGAATTATTTACTTTTTTGTAATTGATTGTAAATTAAATCGGCAAGGCC AAAAAGCTGAGATCTGTCGACATCCATGGTGGCACAGTTTTTGCATGAAAATTAAGTAGT ACTACTTAATTTTCATGCAAAAACTGTGCCACCATGGATGTCGACAGATCTCAGCTTTTT TTTAGTTTAAAACCATTTTTAAATTAAATTGGCACAGTTTTTGCATTTTAACAGGCAAAA TTTTGCCTGTTAAAATGCAAAAACTGTGCCAATTTAATTTAAAAATGGTTTTAAACTAAA TTTAGTTTAAAACCATTTTTAAATTAAATTTTCACAGTTTTTGAATTCCAACAGGCAAAA TTTTGCCTGTTGGAATTCAAAAACTGTGAAAATTTAATTTAAAAATGGTTTTAAACTAAA TCACAATATTCAAGAAAGGGGAGAAAATATAATAACTATGAACATATTTAGTAATGAGGA TCCTCATTACTAAATATGTTCATAGTTATTATATTTTCTCCCCTTTCTTGAATATTGTGA AACATATTTAGTAATGAGGATTTAAACATATATTTAAAATCAGTAAGAGAACACAAGCTA TAGCTTGTGTTCTCTTACTGATTTTAAATATATGTTTAAATCCTCATTACTAAATATGTT TTTAGTTTAAAACCATTTTTAGGCCAAATTTTCACAGTTTTTGAATTCCAACAGGCAAAA TTTTGCCTGTTGGAATTCAAAAACTGTGAAAATTTGGCCTAAAAATGGTTTTAAACTAAA CTCAAAGCTTTGGCCTTGCCGATTTAATTTACAATCAATTACAAAGCTGAGATCTGTCGA TCGACAGATCTCAGCTTTGTAATTGATTGTAAATTAAATCGGCAAGGCCAAAGCTTTGAG GCTGAGATCTGTCGAATCAATTACAAAAAAGTAAATAATTCAAAAAATACTCCCCCTAAA 


\begin{tabular}{|c|c|}
\hline ZM156R & TTTAGGGGGAGTATTTTTTGAATTATTTACTTTTTTGTAATTGATTCGACAGATCTCAGC \\
\hline ZM210F & CTCAAAGCTTTGGCCTTGCCGATTTGGCCTACAATCAATTACAAAGCTGAGATCTGTCGA \\
\hline ZM211F & GCTGAGATCTGTCGAATCAATTACAAAAAAGTAGGCCATTCAAAAAATACTCCCCCTAAA \\
\hline ZM212F & ATTTTATATCCTATTTAGTTTAAAACCATTTTTAAATTAAATTGGCACAGTTTTTGCATG \\
\hline ZM212R & CATGCAAAAACTGTGCCAATTTAATTTAAAAATGGTTTTAAACTAAATAGGATATAAAAT \\
\hline ZM214F & ATTTTATATCCTATTTAGTTTAAAACCATTTTTAGGCCAAATTGGCACAGTTTTTGCATG \\
\hline ZM214R & CATGCAAAAACTGTGCCAATTTGGCCTAAAAATGGTTTTAAACTAAATAGGATATAAAAT \\
\hline ZM215F & TGGTTTAGATTTAATTTTTTTCTCAATTCAGCAATTTCTGAATATAGAATTTTCATATCT \\
\hline ZM215R & AGATATGAAAATTCTATATTCAGAAATTGCTGAATTGAGAAAAAAATTAAATCTAAACCA \\
\hline ZM216F & TGGTTTAGATGGCCTTTTTTTCTCAATTCAGCAATTTCTGAATATAGAATTTTCATATCT \\
\hline ZM218F & AGTGTAAAAAGGCCAATCAATTTTTTCATTAAATATCCTTTTTATTTATTATGGTATTAA \\
\hline ZM218R & TTAATACCATAATAAATAAAAAGGATATTTAATGAAAAAATTGATTGGCCTTTTTACACT \\
\hline ZM223F & ATTTTATATCCTAGGCCGTTTAAAACCATTTTTAGGCCAAATTGGCACAGTTTTTGCATG \\
\hline ZM223R & CATGCAAAAACTGTGCCAATTTGGCCTAAAAATGGTTTTAAACGGCCTAGGATATAAAAT \\
\hline ZM219F & AAAAAAATATGAAAATAAATAAATAATAAGTAGTAAAATATTAATAACTGGGTATAAAAT \\
\hline ZM219R & ATTTTATACCCAGTTATTAATATTTTACTACTTATTATTTATTTATTTTCATATTTTTTT \\
\hline $\mathrm{ZM} 220 \mathrm{~F}$ & AAAAAAATATGAAAATGGCCAAATAATAAGTAGTAAAATATTAATAACTGGGTATAAAAT \\
\hline ZM220R & ATTTTATACCCAGTTATTAATATTTTACTACTTATTATTTGGCCATTTTCATATTTTTTT \\
\hline
\end{tabular}

